\section{Corrections to Two Previous Articles on Extragalactic Gamma Rays and Implications of the Corrected Results}

I REPORT here the correction of an error which, unrortunately, affects two articles of mine on extragalactic gamma radiation which appeared recently in Nature ${ }^{1,2}$. Equation (1) of ref. 1 should contain a factor of $(1+z)^{2}$ in the denominator instead of $(1+z)^{3}$. The same correction, applied to equation (12) of ref. 2, affects the index, $\alpha$, of equation (15). This index then becomes 2 for a low density model and 1.5 for an Einstein -de Sitter model universe

The change in $z$-dependence of equation (1) in ref. 1 lowers the requirements on the present metagalactic cosmic-ray intensities given in Tables 1 and 2 of that article. The corrected values are given in this article.

\begin{tabular}{|c|c|c|c|c|c|c|}
\hline \multirow[b]{2}{*}{ Model } & \multicolumn{6}{|c|}{ Table 1. VALUK OF $I_{O} / I_{\sigma}$ FOR $n_{0}=10^{-5} \mathrm{~cm}^{-3}$} \\
\hline & $z_{\max }$ & $2 \cdot 2$ & 4 & 9 & 40 & 100 \\
\hline \multicolumn{2}{|c|}{$\begin{array}{l}\text { Constant leakage } \\
\text { Burst model } \\
\text { Erolving sources }\end{array}$} & $\begin{array}{l}1.8 \times 10^{-1} \\
2.6 \times 10^{-2} \\
2.5 \times 10^{-2}\end{array}$ & $\begin{array}{l}1.3 \times 10^{-1} \\
9.0 \times 10^{-8} \\
6.0 \times 10^{-3}\end{array}$ & $\begin{array}{l}7.0 \times 10^{-2} \\
2.2 \times 10^{-3} \\
4.8 \times 10^{-4}\end{array}$ & $\begin{array}{l}3.2 \times 10^{-8} \\
3 \cdot 0 \times 10^{-4} \\
7 \cdot 1 \times 10^{-6}\end{array}$ & $\begin{array}{l}2.8 \times 10^{-2} \\
1 \cdot 1 \times 10^{-4} \\
4 \cdot 1 \times 10^{-7}\end{array}$ \\
\hline \multicolumn{7}{|c|}{ Table 2. VALUR OF $I_{o} / I_{g}$ FOR $n_{\theta}=10^{-7} \mathrm{em}^{-2}$} \\
\hline Model & \multicolumn{2}{|c|}{$z_{\max } 2 \cdot 2$} & 4 & 9 & 40 & 100 \\
\hline $\begin{array}{l}\text { Constant leaka } \\
\text { Rurst model } \\
\text { Evolving sourc }\end{array}$ & \multicolumn{2}{|c|}{$\begin{array}{l}13 \\
1 \cdot 7 \\
1 \cdot 6\end{array}$} & $\begin{array}{c}7 \cdot 2 \\
4.9 \times 10^{-1} \\
2 \cdot 4 \times 10^{-1}\end{array}$ & $\begin{array}{c}2 \cdot 3 \\
8.9 \times 10^{-2} \\
1.0 \times 10^{-2}\end{array}$ & $\begin{array}{l}3.6 \times 10^{-1} \\
6.5 \times 10^{-3} \\
1.4 \times 10^{-4}\end{array}$ & $\begin{array}{l}2.0 \times 10^{-1} \\
1.6 \times 10^{-3} \\
1.3 \times 10^{-5}\end{array}$ \\
\hline
\end{tabular}

The values given in Stecker and Silk ${ }^{3}$ are correct.

This same correction changes the results given in Figs. 2 to 5 of ref. 2 . The power-law spectra generated then have steeper slopes, as shown in the corrected figures presented here (Figs. I to 4). The implication of this correction is more profound.

The Harrison cosmology ${ }^{4}$ considered in ref. 2 leads to an estimate that $\sim 1^{-9}$ of the original mass of the universe remains after the initial annihilation of almost all of the initial matter and antimatter in producing the thermal blackbody radiation. To order of magnitude then, the photon density of annihilation-gamma-radiation cannot be more than $10^{-9}$ of the photon density of the blackbody radiation; that is, about $6 \times 10^{-7} \mathrm{~cm}^{-3}$. Using this density as an upper limit, and assuming the annihilation-gamma-ray spectrum follows a power law form of

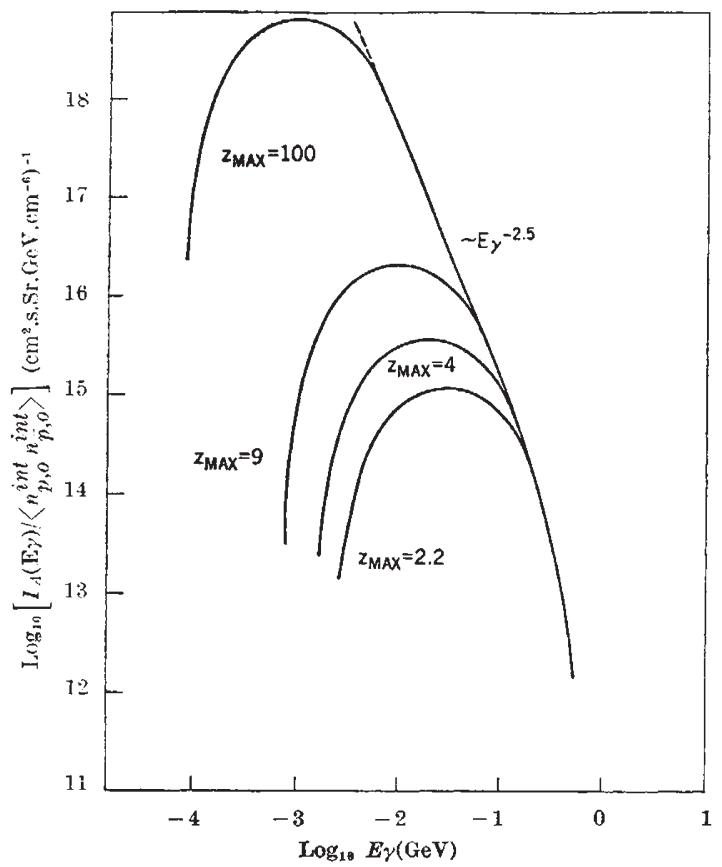

Fig. 1. The metagalactic differential annihilation spectrum given for various maximum red-shifts for an Einstein-de Sitter universe.

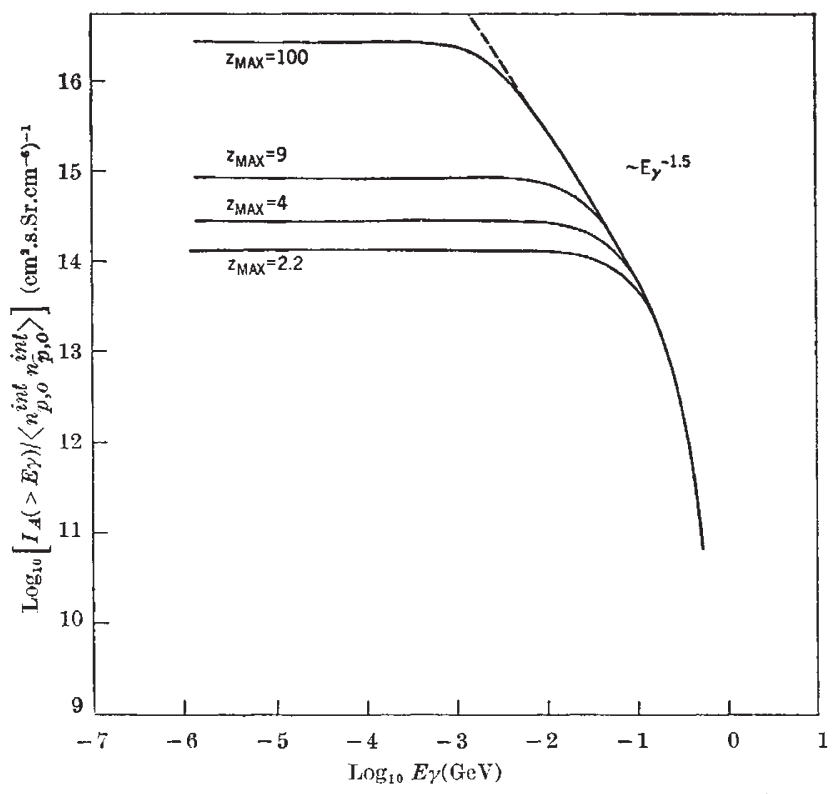

Fig. 2. The metagalactic integral annihilation spectrum given for various maximum red-shifts for an Einstein-de Sitter universe.

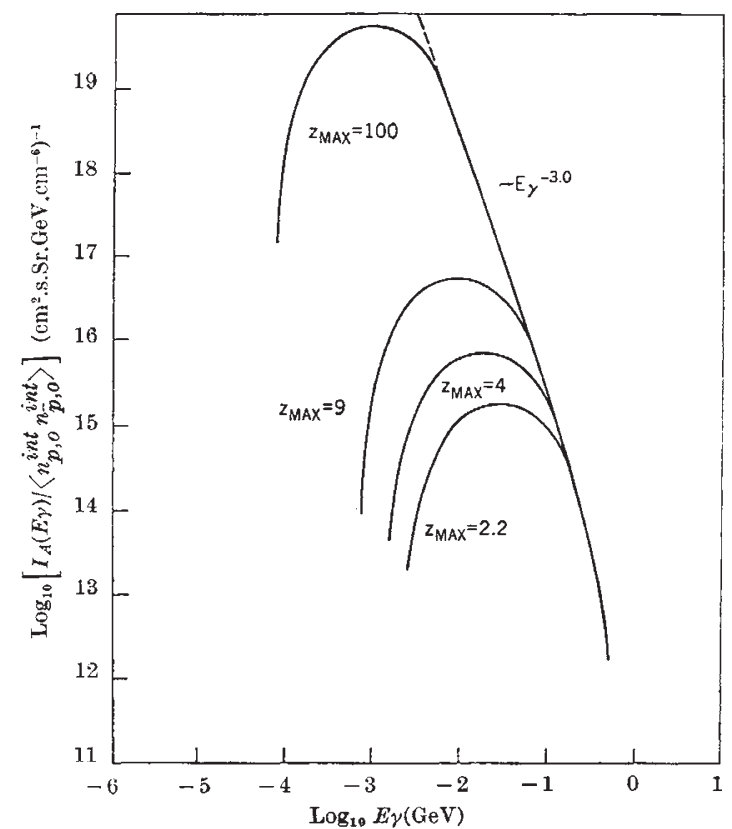

Fig. 3. The metagalactic differential annihilation spectrum given for various maximum red-shifts for a low density universe.

$I(E)_{\gamma} \mathrm{d} E_{\gamma}=K E_{\gamma}{ }^{-} \zeta \mathrm{d} E_{\gamma}$, we find that if this spectrum is to match up with the intensity of the observed X-ray spectrum at $100 \mathrm{keV}$, then $\xi \leq 1.5$. A larger value of $\xi$ would result in an annihilation-photon density much too high. Coincidentally, a value for $\xi$ of 1.5 seems to fit the data well in the range below $100 \mathrm{keV}$ (and possibly up to $\mathrm{I} \mathrm{MeV}$, as will be shown elsewhere by Dr Trombka of our branch). Unfortunately, when the error in ref. 2 is corrected we obtain values of 2.5 and 3.0 for the Einstein-de Sitter model and the low-density model respectively and these values are too high to account for an annihilation interpretation of the X-ray data. Perhaps a more general cosmological model could yield the stronger curvature factor needed, namely, $\mathrm{d} l / \mathrm{d} z \simeq c H_{0}^{-1}(1+z)^{-3.5}$ for $10^{2} \leq z \leq 10^{4}$, to give a value for $\xi$ of 1.5 . Also, one 


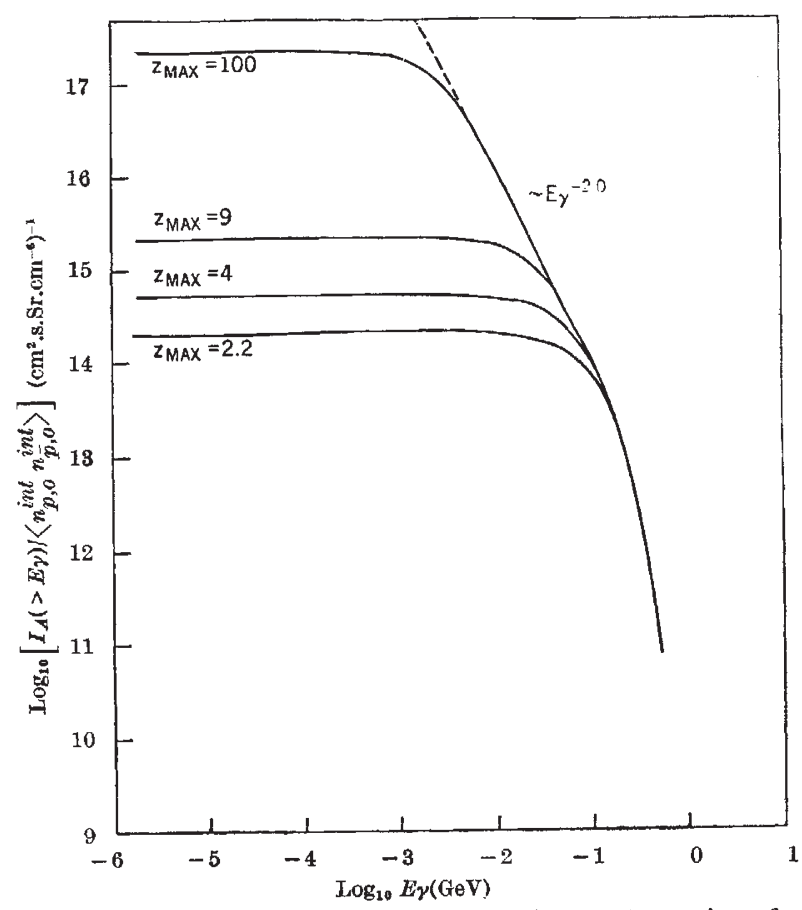

Fig. 4. The metagalactic integral annihilation spectrum given for various maximum red-shifts for a low density universe.

should consider the temperature dependence of the atomic annihilation rate for $z$ is $\leq 10^{4}$.

Theoretical Studies Branch,

F. W. STECkER

Goddard Space Flight Center,

Greenbelt, Maryland.

Received February 24, 1969.

'Stecker, F. W., Nature, 220, 675 (1968).

2 Stecker, F. W., Nature, 221, 425 (1969).

Stecker, F. W., and Silk, J., Nature, 221, 1229 (1968).

4 Harrison, E. R., Phys, Rev. Lett., 18, 1011 (1967).

\section{Search for Hydrogen appearing in Mercury Metal}

Twenty years have passed since Bondi and Gold ${ }^{1}$ introduced the steady-state theory of the universe and the attendant concept of continuous creation. Thus far, the theoretical successes of steady-state cosmology have survived the tests of observation ${ }^{2}$. In all of the observations, however, there has been no direct evidence for or against continuous creation.

In the original form of the steady-state theory ${ }^{1}$, the creation rate is proportional to the proper volume and takes place at such a rate that the mean density of matter in the universe does not undergo secular change, despite the expansion determined from the red-shift. This rate can be shown to be $3 \rho / T$ (ref. 3 ), where $p$ is the mean density of matter in the universe, and $T$ is Hubble's constant. If we take $p=2 \times 10^{-29} \mathrm{~g} / \mathrm{cm}^{3}$ and $1 / T=2.4 \times 10^{-18} \mathrm{~s}^{-1}$, the rate is $1.4 \times 10^{-46} \mathrm{~g} / \mathrm{cm}^{3} \mathrm{~s}$. This, as Bondi pointed out ${ }^{4}$, cannot be observed directly. If, however, one makes the assumption that continuous creation is proportional to the amount of mass in a volume, rather than the volume itself, then the rate ${ }^{3}$ would be $7 \cdot 2 \times 10^{-18} \mathrm{~g} / \mathrm{g} / \mathrm{s}$.

We do not know in what form newly created matter would appear, but hydrogen, as the most abundant element, seems a likely possibility. Unlike charged pairs, it would not generate radiation on a cosmic scale in violation of present observational limits.

In the spring of 1968 , we performed an experimental test of mass-proportional creation of hydrogen. To trap and separate the hydrogen from the bulk matter within which it would be created, we chose the simple processes of freezing and boiling mercury. Distillation of 1 mole of mercury would release $9 \times 10^{8}$ hydrogen atoms for each second during which the mercury was in the frozen state. For our $0.6 \mathrm{l}$. volume this corresponds to a pressure increase of $4 \times 10^{-11}$ torr $/ \mathrm{s}$.

The apparatus was an inverted Y-tube (Fig. 1). The mercury was alternately distilled from one leg to the other. In the stem of the $\mathbf{Y}$-tube were three magnetic stainless-steel ball valves and a liquid nitrogen trap. The stem of the $Y$-tube was connected to an omegatron mass spectrometer and to a pumping system that maintained a pressure of $5 \times 10^{-8}$ torr or less. During distillation the pumping system was separated from the omegatron and Y-tube by a vacuum valve. The mercury was then distilled from one leg of the $\mathrm{Y}$ into the other where it was condensed and frozen. The ball valves were then opened to allow any gases evolved to pass into the omegatron.

The omegatron was calibrated by leaking known amounts of hydrogen into the apparatus and observing the response of the omegatron. This calibration agreed with the calculated sensitivity of the omegatron within a factor of two, Because the omegatron dissociates $\mathrm{H}_{2}$ into $\mathrm{H}^{+}$, as well as $\mathrm{H}_{2}^{+}$, two peaks were observed the heights of which were in the ratio $\mathrm{H}_{2}^{+} / \mathrm{H}^{+}=1.39$; only the $\mathrm{H}_{2}^{+}$peaks were recorded during the runs. The ion pump action of the omegatron was entirely negligible for the amount of gas to be observed. The system was surrounded by $\mathrm{H}_{2}$-filled bags for leak testing, and no leakage was found. Also, the possibility that the hydrogen was being lost in the mercury was eliminated by experi. ments in which hydrogen was dissolved in the mercury and measured in successive distillations.

The amount of $\mathrm{H}_{2}$ evolved in each distillation, as well as the amount evolved when the empty leg of the $Y$ was heated, was measured. The outgassing pressure of the vycor walls averaged, over a dozen runs, to $4 \times 10^{-10}$ torr for a time of $30 \mathrm{~min}$ at a temperature of $300^{\circ} \mathrm{C}$. This was subtracted from the observed pressure to yield the amount of hydrogen that evolved from the mercury alone. The

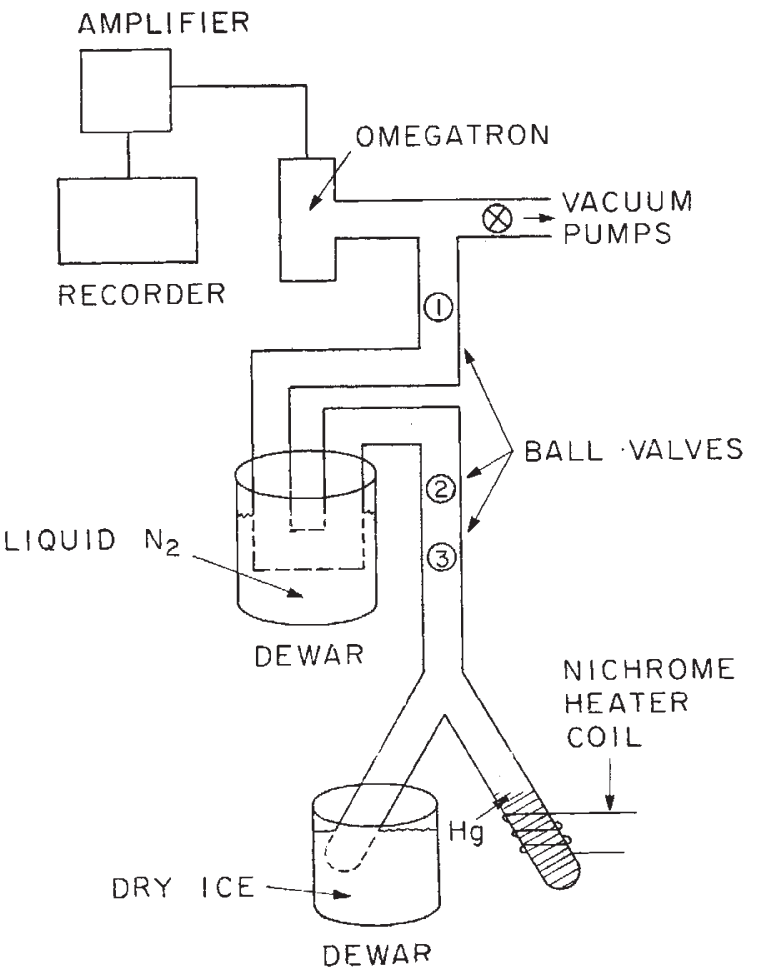

Fig. 1. The apparatus. 